\title{
Hybrid MIMO: A New Transmission Method For Simultaneously Achieving Spatial Multiplexing and Diversity Gains in MIMO Systems
}

\author{
JOEL PONCHA LEMAYIAN ${ }^{1}$, JEHAD M. HAMAMREH ${ }^{\mathbf{1}}$ \\ ${ }^{1}$ WISLAB, Department of Electrical and Computer Engineering, Antalya Bilim University,Antalya, Turkey (e-mail: lemayian.joel@ std.antalya.edu.tr) \\ ${ }^{1}$ WISLAB, Department of Electrical and Electronics Engineering, Antalya Bilim University,Antalya, Turkey (e-mail: jehad.hamamreh@antalya.edu.tr) \\ Corresponding author: J. P. Lemayian and J. M. Hamamreh (emails: lemayian.joel@std.antalya.edu.tr, jehad.hamamreh@gmail.com, Web: \\ https://sites.google.com/view/wislab).
}

This work is funded by the scientific and technological research council of Turkey (TÜBITAK) under project grant No. 119E392. All the codes used can be found at researcherstore.com

\begin{abstract}
Multiple input multiple output (MIMO) technology has evolved over the past few years into a technology with great potential to drive the direction of future wireless communications. MIMO technology has become a solid reality when massive MIMO systems (MIMO with large number of antennas and transceivers) were commercially deployed in several countries across the world in the recent past. Moreover, MIMO has been integrated into state-of-the-art paradigms such as fifth-generation (5G) networks as one of the main enabling technologies. MIMO possesses many attractive and highly desirable properties such as spatial multiplexing, diversity gains, and adaptive beamforming gains that leads to high data rates, enhanced reliability, and other enhancements. Nevertheless, beyond 5G technologies demand wireless communication systems with, among other properties, immensely higher data rates and better reliability simultaneously at the same time. In this work, a new, novel MIMO technique for simultaneously achieving multiplexing and diversity gains as well as completely eliminating any processing at the MIMO receiver, leading to advantages such as low complexity and low power consumption, is proposed. The proposed technique employs the design of interference-canceling matrices, which are calculated from the channels between the transceiver antennas, where the matrices are employed at the base station to help achieve multiplexing and diversity gains simultaneously. The novelty and efficiency of the introduced paradigm is demonstrated via mathematical models and validated by Monte Carlo simulations. Results indicate that the proposed system outperforms conventional MIMO models.
\end{abstract}

INDEX TERMS Multiple input multiple output, MIMO, Massive MIMO, internet of things (IoT), low power, low complexity, spatial multiplexing, diversity gain.

\section{INTRODUCTION}

Multiple input multiple output (MIMO) technology has highly desirable properties such as high throughput and high spectral efficiency, which are enabled by its spatial multiplexing mode; and better reliability, which is enabled by its diversity mode [1] [2]. It has evolved from a mere research concept to a real-world technology and has been integrated into state-of-the-art wireless network standards such as IEEE 802.11n, 3GPP long-term evolution (LTE), mobile WiMAX systems, LTE-Advanced (E-UTRA), and recently in $5 \mathrm{G}$ systems. Due to the exponential increase in the number of connected things, the ability of MIMO to have high user capacity is a key requirement for the current 5GIoT era and beyond technologies (6G). MIMO has the ability to provide enhanced throughput even under conditions of interference, signal fading, and multipath. The demand for wireless communication services is continuously increasing as a consequence of the massive spread of wireless devices featured by high mobility and ease of use. Additionally, the surge in wireless data communication is primarily driven by the huge number of beneficial applications customized for mobile users. For instance, the Internet of things (IoT) 
is a network of millions of interconnected wireless devices accessible through the internet. The idea of IoT was made possible by advanced wireless communication technologies ( $5 \mathrm{G}$ and beyond). This is due to the many advantages such as increased data rate, reduced delay, and enhanced cellular coverage in the communication technologies over preceding technologies. These advantages will have a huge impact on future intelligent service delivery. Some areas influenced by IoT are autonomous driving, healthcare, entertainment, industrial appliances, smart cities, smart energy grids, sports, remote surgery, and drone delivery applications. Moreover, countries around the world are employing IoT technologies to combat challenges such as traffic congestion, insecurity, and infrastructure management caused by overpopulation. MIMO systems are used in IoT networks to increase data rate and diversity. Therefore, MIMO has set its self as one of the leading technology that will continue to shape the future of wireless communications.

\section{A. TECHNICAL REVIEW}

Three key techniques are employed in standard MIMO systems to enhance its performance, these techniques are spatial multiplexing, space-time block coding (STBC), and beamforming. Spatial multiplexing (SM) is a wireless communication technique that allows many antennas to simultaneously send multiple streams of data to many receivers using the same frequency and time resources [3]. The receiver then decode each stream of data to reconstruct the original transmitted data. Spatial multiplexing increases data rate, nevertheless, the bit error rate (BER) is not very reliable because the streams of data interfere with each other.

STBC is a MIMO technique where multiple copies of a data stream are sent via the multiple transmit antennas and then exploiting the many receive antennas to receive the different versions of the signal which improves the reliability of data transferred [4]. STBC combines all the received versions in an optimal way to extract maximum diversity from all the received signals. Since STBC involves the transmission of multiple copies of the same signal, it helps to reduce channel degradation caused by fading and thermal noise, therefore, some copies of the received signal may be less corrupted than others. In STBC, the data streams are first encoded in blocks before transmission. The data blocks are then distributed across the different antennas and the data is also spread out across time. An STBC model is usually represented by a matrix, shown in (1), where each element in the row represents a time slot while each element in the column represents a single antenna's transmission over time. Therefore, in the STBC matrix, the element, $S_{i j}$, is the modulated symbol to be transmitted in time slot $i$ from antenna $j$. STBC helps to reduce error at the receiver, however, the data rate performance in its best cases is similar to a system utilizing a single input single output (SISO) technique, where the scheme achieving such performance is named in the literature as a full-rate STBC scheme.

$$
\left[\begin{array}{cccc}
\mathbf{S}_{11} & \mathbf{S}_{12} & \cdots & \mathbf{S}_{1 \mathrm{n}} \\
\mathbf{S}_{21} & \mathbf{S}_{22} & \cdots & \mathbf{S}_{2 \mathrm{n}} \\
\cdot & \cdot & \cdot & \cdot \\
\cdot & \cdot & \cdot & \cdot \\
\mathbf{S}_{\mathrm{m} 1} & \mathbf{S}_{\mathrm{m} 2} & \cdots & \mathbf{S}_{\mathrm{mn}}
\end{array}\right] .
$$

The third mode of MIMO is called beamforming, which is a wireless communication technique in which signals from transmit antennas are focused towards the receiver rather than broadcasting the signals in all direction as conventionally done [5] (See Fig. 2). To achieve beamforming, a single symbol is usually sent from all transmit antennas at slightly different times by changing their phases in such away that they add constructively at the receiver side. The resulting direct signal connection is faster and more reliable. While beamforming technique was introduced in 1940s, it has gained momentum in recent years especially in Wi-Fi networking and 5G systems; for instance, current beamforming methods use multiple vertical and horizontal arrays of antennas put together to form a 3D beam focusing the signal to a user equipment (UE) in any direction. As a result, beamforming technology is a key enabling technology of the current 5GIoT systems. In beamforming technique a single symbol is sent from all the antennas, hence, this technique has good signal to noise ratio (SNR) but bad data rate.

Using the techniques mentioned above, MIMO is set to provide the following advantages to wireless communications:

1) Spatial multiplexing gain: This is the ability of MIMO systems to use multiple streams to transmit data to users, it leads to enhance capacity and peak throughput.

2) Diversity gain: MIMO systems achieve diversity gain by sending data signals to user equipment through multiple streams (multipath).

3) Interference reduction: Beamforming can be used to form narrow beams towards users, thus reducing interference among users and improving the signal-tointerference-plus-noise ratio (SINR); moreover, coverage and capacity are also improved.

4) Array gain: This is the increase in SNR due to multiple antennas transmitting the same signal in different phases, sometime called beamforming gain.

\section{B. MOTIVATION}

Current MIMO systems and enabling technologies do not meet the requirements and demands of future wireless communications. For instance, IoT and massive machine type communications (mMTC), which are in no doubt the epitome of current technological progression, demand communication technologies with lower power and complexity requirements. For example, a receiver designed for future IoT wireless devices must be very simple to meet the low power and low complexity demands due to the small size of the devices. Nevertheless, technologies utilized in current MIMO 


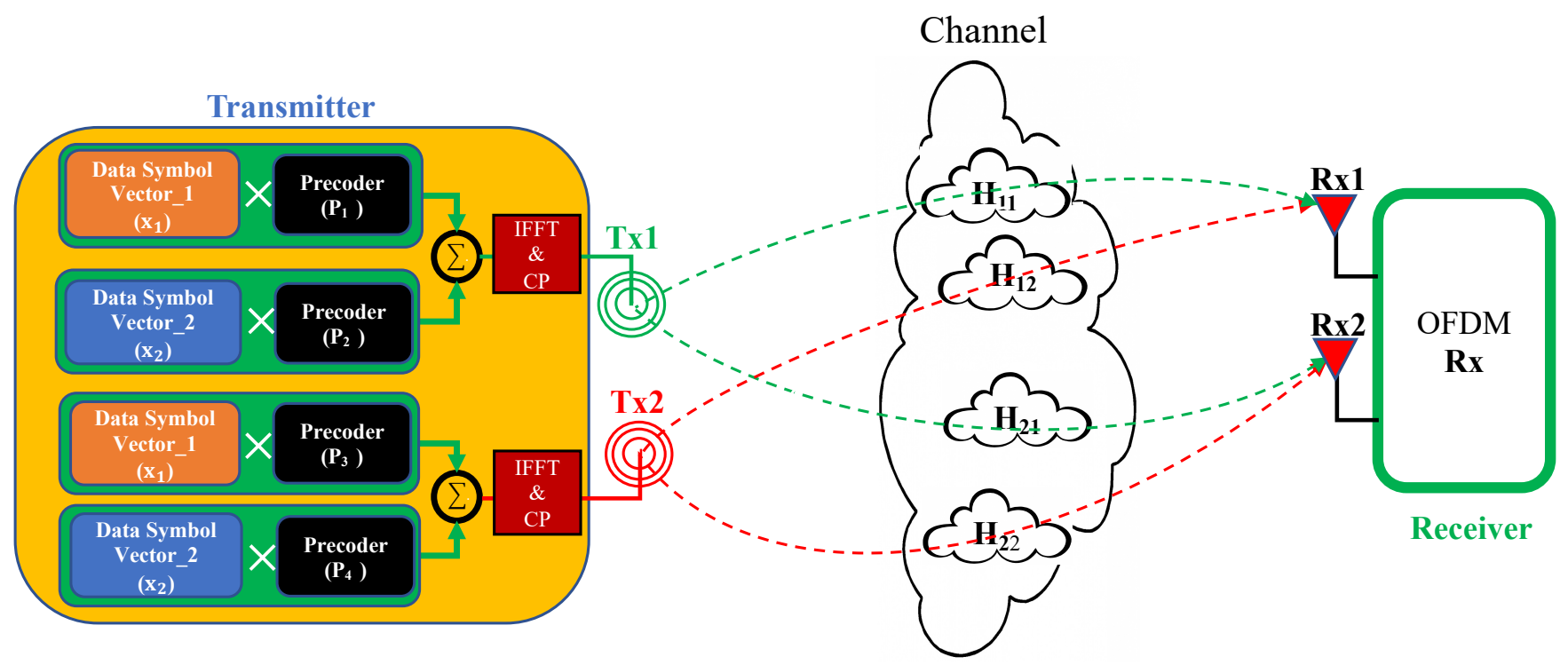

FIGURE 1. Proposed multiple input multiple output (MIMO) system utilizing physical layer security. The MIMO system uses two antennas but can be expanded to use many antennas.

systems such as zero forcing (ZF) equalization, vertical-bell laboratories layered space-time (V-BLAST), minimum mean square error (MMSE) equalization, zero forcing equalization with successive interference cancellation (ZF-SIC), and maximum likelihood (ML) decoding add more complexity to the receivers.

Next generation wireless communication applications also demand communication systems with higher reliability, lower latency, and higher data rate. Such applications include extended reality (XR), virtual reality (VR), telemedicine, brain-computer interface, and more [6]. Therefore, novel communication systems with the ability to meet such demands must be realized.

Hence, there is a strong demand for a single wireless MIMO communication scheme which can combine and fuse all the advantages provided by individual MIMO techniques including space-time coding, beamforming, and spatial multiplexing to better meets the demands of low complexity, high throughput, and ultra-reliable receiver design for future mMTC and IoT devices.

Fig. 1 shows the system model of the proposed model. This technology can be implemented for many transceivers, however, a system with two transmitters and two receivers is analysed in this work for simplicity purposes. From the figure, it can be observed that interference-canceling matrices are multiplied by user data before superimposition and transmission. Also, a single user with two antennas can be observed, where the user simply received the transmitted data without performing any processing leading to advantages such as low complexity and low power consumption.

\section{NOVELTY OF THE PROPOSED MODEL}

Current and future wireless communication technologies demand communication systems with all the advantages of the three MIMO communication techniques, in other words, a wireless communication system with increased data rate, reduced BER, and signal focusing capabilities with low complexity detection process at the IoT receiver is needed for current and future massive wireless communication paradigms. In this work, we propose a novel MIMO communication technique for achieving multiplexing and diversity gains simultaneously while making sure no complex processing is done at the receiver side. This novel MIMO transmission scheme (shown in Fig. 1) uses interference-canceling matrices to prevent inter-symbol interference. In the figure, multiple data symbol vectors are superimposed and simultaneously transmitted from all the antennas. Each frequency domain data symbol vector in an OFDM-MIMO system is first precoded by an interference-canceling matrix before superposition. The proposed Hybrid MIMO technique ${ }^{1}$ has the following properties:

1) Increased data rate: Multiple superimposed symbols are send from each antenna at a given time.

2) Reduced BER: Each data symbol vector is send from many different antennas at the same time.

3) Beamforming capabilities: The same superimposed signal with interference-canceling matrices is transmitted from many different antennas, therefore, the transmission can be manipulated to perform beamforming towards the MIMO receiver as shown in Fig. 2.

\footnotetext{
${ }^{1}$ It is worth mentioning that the proposed novel design in this paper has significantly been inspired by the authors' previous studies, mainly [7][10], which were applied to different domains and intended for addressing different problems.
} 
4) Low complexity: The proposed system is designed such that the receiver simply receives the data without any processing, where all the signal processing and computations are done at the transmit base station whose power is usually not limited. Therefore, this communication technique is perfect for IoT applications with limited power and processing capabilities.

Fig. 2 shows the proposed technique where the interference-canceling or fading-controlling matrices $\left(\mathbf{P}_{\mathbf{1}}, \mathbf{P}_{\mathbf{2}}, \mathbf{P}_{\mathbf{3}}\right.$ $\left.\mathbf{P}_{4}\right)$ are used to prevent inter-symbol interference and control/mitigate fading at the receiver, the multiple antennas are utilized to achieve beamforming towards the UE. In this work, a two antennas design is implemented as shown in Fig. 1 and Fig. 2 for simplicity purposes; however, this design can be extended for any number of antennas with potential use to multi-user scenarios.

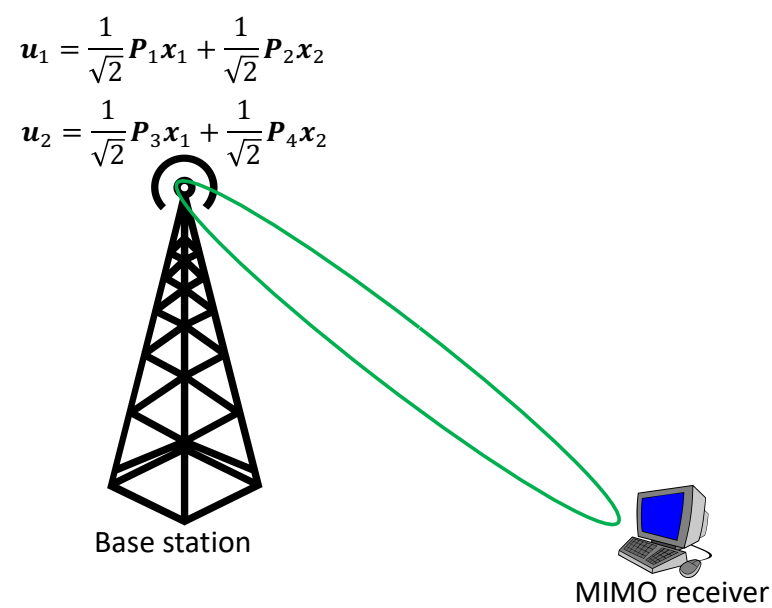

FIGURE 2. The proposed model can also be designed to perform beamforming, which leads to enhanced security, increased reliability, better cell range, high capacity, and improved spectral efficiency.

\section{LITERATURE REVIEW}

As a technology with high potential, multiple works have been done in literature and the industry to try and enhance the performance of MIMO systems. As stated in the previous sections, MIMO can be classified into three distinct technologies [11]: spatial multiplexing, space-time block coding (STBC), and beamforming. Each of these technologies have strengths and weaknesses. Spatial multiplexing has increased data rate but bad BER, STBC has good BER but bad data rate, and beamforming has good SNR but bad data rate. Therefore, current technology is trying to achieve all the benefits of these techniques and reduce their drawbacks using a single technology.

Authors in [12] propose an STBC with spatial multiplexing for MIMO transmission. According to the authors, the invention provides the STBC-based communication with uniform protection on all data streams such that the data streams experience high SNR, consequently the combined STBC and spatial multiplexing results in enhanced performance such as high throughput WLANs (e.g., IEEE 802.11n). The MIMO system comprises of a transmitter with an STBC encoder that encodes $N_{s s}$ input data streams using STBC for communication through $N_{t}$ transmit antennas. When $N_{t}<2 N_{s s}$ the network simultaneously performs $N_{t} N_{s s}$ encoding operations to $N_{s s}$ input data streams.

Moreover, authors in [13] propose a combination of STBC with spatial multiplexing technique applied to MIMO to take advantage of both techniques. In the proposed technique, the transmit antennas are divided into two, and each antenna communicates space-time coded blocks in parallel. Furthermore, the receiver is comprised of three groups, the first part filters the received signal, the second part separates the filtered multiplexed space-time coded symbol blocks, and the third part is a space-time decoder. According to the authors, results indicate that the proposed system has larger diversity than spatial multiplexing system with the same data rate as the proposed system. Moreover, the data rate of the combined STBC and special multiplexing is larger than STBC system only.

Authors in [14] propose a low-complexity optimal detection for hybrid STBC and spatial multiplexing. According to the authors, this combination has the advantages of diversity gain and high data rate compared to the individual techniques. Authors utilize a novel detector based on the block structure of STBC and a sphere decoder (SD). Moreover, authors also claim that using the SD provides for optimal sensing as well as lower computation complexity compared to maximum likelihood (ML). According to the authors, results obtained indicate that the proposed system outperforms previously developed zero-forcing (ZF) group/STBC detector, the minimum mean square error (MMSE) group/STBC detector, and the MMSE and ML detectors for equivalent SM systems.

Authors in [15] propose a novel high-rate distinction STBC technique based on spatial multiplexing of Alamoutiencoded information sequence. The system is composed of two symbols and two transmit antennas of a flat-fading channel. Moreover, space-time differential interference cancellation and decoding are jointly performed at the receiver without prior information of the channel or the need for extra bandwidth, leading to diversity and high data rate.

Likewise, authors in [11] consider STBC and spatial multiplexing MIMO techniques as methods of enhancing the performance of coded orthogonal frequency division multiplexing (COFDM) wireless local area networks (WLANs). According to the authors, square channel estimator was utilized to achieve high system performance. Moreover, the $2 \mathrm{Rx}-$ 2Tx antenna system exhibits high throughput performance, authors claim that STBC has twice the throughput in low SNR values. In addition, authors claim that the proposed multiplexed technique achieves better throughput performance at high SNR values at the expense of increased complexity.

Authors in [16] propose a MIMO OFDM system consisting of space-time encoders for encoding independent spacetime coded data blocks. The data signals are sent and 
received by numerous antennas and are denoised before maximum likelihood detection. According to the authors, successive interference cancellation (SIC) is used to enhance the performance of the system. Moreover, the estimation of channel parameters can be improved by taking into account the deviation of the channel impulse response from the average when calculating it.

Authors in [17] propose a MIMO communication device which can maintain a MIMO communication system's characteristic or more at a given level, regardless of the installation position of the device. The system is composed of multiple antennas with a MIMO modulation unit connected to each antenna, this guarantees that there exists at least one combination of elements with a channel estimation matrix of the channel between the MIMO communication device and the user regardless of the installation location of the MIMO device. Moreover, authors in [18] designed a MIMO communication system with beamforming using polar-cap codebooks.

Authors in [19] implemented blind turning in hybrid MIMO RF beamforming systems. The system applies the blind turning process to $M$ antennas consisting of $N$ beamformers to a MIMO receiving system consisting of $N$ channels, where $M>N$. The proposed method consists of the following steps: 1) The channel fading rate is periodically determined at baseband level, so as to determine the number of antennas $L$ out of $M$ connected to the beamformers. 2) A selection criterion such as best quality indicator is used to assign the antennas to the subset $L$, and 3) Applying a tuning process to the $L$ antennas in all the beamformers. Moreover, authors in [20] designed systems and methods for uplink MU-MIMO medium access and error recovery.

Authors in [21] implement a method for maximizing MIMO's throughput by deploying antennas in a vehicle. According to the authors, the arrangement of antennas in the vehicle will include a primary MIMO antenna that may be used for non-4G cellular services, as well as one secondary MIMO antenna that may be used only for $4 \mathrm{G}$ cellular services. In one setting, the primary antenna may be placed on the roof-top of the vehicle with other antennas, and one or more secondary antennas maybe placed inside the vehicle at a desired position. In another setting, all the antennas, including the primary, secondary, and other antennas are placed inside the vehicle.

Authors in [22] propose using antenna pooling to enhance a MIMO receiver and further improved by RF beamforming. The system has a multilayer MIMO receiving paradigm, a MIMO baseband unit, and a radio distribution network (RDN) linked to the MIMO receiving system. The RDN consists of two or more beamformers connected to two or more antennas such that the total number of antennas in the system is greater than the number of branches in the MIMO baseband unit, moreover, each of the beamformers combine the RF signal from the antennas. Furthermore, the system implements an antenna routing technique where a set of quality indicators are considered to switch the antennas between different beamformers, hence, this increases the probability of grouping together antennas with minimum phase interference from the transmitted signals. According to the authors, the system increases the range of antenna selection above the available set of antennas on each beamformer. Moreover, authors in [23] propose systems and methods for combining MIMO and mode-division multiplexing, while authors in [24] propose a technique to increase a code rate in a MIMO system using virtual channels.

In [25], authors investigated schemes that combine two or three techniques and the schemes can be considered alternatives to each other. For example, multifunctional MIMO, combines the benefits of several MIMO schemes including diversity gains, multiplexing gains, and beamforming gains. Moreover, a combination of V-BLAST which is capable of achieving the maximum attainable multiplexing gain and STBC which can attain the maximum achievable antenna diversity gain was proposed in [26] to provide both antenna diversity and spectral efficiency gains. In another technique to achieve additional performance gains, beamforming has been combined with both spatial diversity as well as spatial multiplexing techniques. Also, STBC has been combined with beamforming to attain an improved SNR gain in addition to the diversity gain [27] [28].

Inspired by the previous three schemes, El-Hajjar et al. [29] proposed a tri-functional MIMO scheme that combines diversity gain with multiplexing gain and beamforming gain, this scheme is called layered steered space-time code (LSSTC), where the parallel data streams are encoded by OSTBC layers, and each layer has a different OSTBC structure. Additionally, the decoder of the LSSTC scheme employed an ordering strategy for decoding the different layers to improve the achievable performance. This scheme is proposed for different numbers of antennas at the transmitter and receiver side. However, the two sides must have an equal number of antennas for interference cancellation to work. To serve more users, with the same concept as in LSSTC, a new technique was introduced in [29] named layered steered space-time spreading (LSSTS). The LSSTS scheme combines the benefits of V- BLAST, STS, and beamforming with generalized multicarrier direct sequence CDMA (MC DS-CDMA) for obtaining multiplexing gain, spatial and frequency diversity gains, and beamforming gain. This scheme includes 4 transmit antennas as well as 2 receive antennas and a linear receiver to decode the received signal.

Therefore, the design of the multifunctional MIMO scheme will depend on the application, for a handheld device the LSSTC scheme is not a practical choice because of the limited size and complexity of the small device. On the other hand, the LSSTS scheme can be considered a good choice for a system with a small-sized receiver that requires high robustness and throughput [25]. Although the LSSTC and LSSTS schemes combine the three techniques to achieve higher data rate, diversity gain, and beamforming gain, they still fall short in terms of more reliability and the huge demand for higher data rates for an increasing number 
of users as well as IoT devices. There is no concept of superpositioning of the signals nor increasing the reliability of the signals even further. beamforming interference can be minimized, but due to the randomly varying nature of the transmission medium it becomes difficult to allocate proper resources to ensure user connectivity. Moreover, security is still a big issue, beamforming facilitates some confidentiality but if the eavesdropper can get close enough to the user the information can easily be leaked, this has become a major concern in the current IoT devices and for future systems as well.

The remainder of this work is composed of the following sections: Section II briefly discusses an overview of the MIMO system model, section III discusses the proposed algorithm. Section IV discusses the performance analysis methods and section $\mathrm{V}$ discusses the simulation results. Section VI discusses the main advantages of the proposed technology, section VII discusses the main limitations, and finally, Section VII discusses the conclusion and future works.

\section{Nomenclature}

AN : Artificial Noise

AWGN : Additive white Gaussian noise

BER : Bit Error Rate

COFDM : Coded Orthogonal Frequency Division Multiplexing

E - UTRA : Evolved Universal Terrestrial Radio Access

EAP : Extensible Authentication Protocol

LTE : Long-Term Evolution

MIMO : Multiple Input Multiple Output

ML : Maximum Likelihood

MMSE : Minimum Mean Square Error

mMTC : massive Machine-Type Communications

MRC : Maximum Ratio Combining

OFDM : Orthogonal Frequency Division Multiplexing

OOBE : Out-Of-Band Emission

PAPR : Peak to Average Power Ratio

PD - NOMA : Power Domain Non-Orthogonal Multiple Access

PLS : Physical Layer Security

PSaaS : Physical Security as a Service

RDN : Radio Distribution Network

SD : Sphere Decoder

SISO : Single Input Single Output

STBC : Space-Time Block Coding

TDD : Time Division Duplexing

TTLS : Tunneled Transport Layer Security

UAV : Unmanned Aerial Vehicles

UE : User Equipment

URLLC : Ultra-Reliable Low-Latency Communications

WEP : Wired Equivalent Privacy

WLAN : Wireless Local Area Network

WPA : Wi-Fi Protected Access

ZF : Zero-Forcing

\section{THE PROPOSED MIMO SYSTEM MODEL}

The proposed MIMO technique can be designed to utilize any number of transmit and receive antennas in a MIMO system; nevertheless, for simplicity and clarity purposes, this work proposes a design where only two transmit and two receive antennas are used in an OFDM-MIMO system. In the proposed system, a MIMO transmitter with two antennas is trying to communicate with a MIMO receiver with two receivers as shown in Fig. 1. In the figure, the frequency channel response is considered to be slowly varying multipath Rayleigh fading and exponentially decaying. The channel is indicated by $\mathbf{H}_{k m}$, with size $N_{f}$ by $N_{f}$, where $\mathbf{H}_{k m}$ is the diagonal channel frequency response between transmit antenna $m$ and receive antenna $k(k=[R x 1, R x 2])$ in an OFDM system with $N_{f}$ number of subcarriers.

\section{PROPOSED ALGORITHM}

This work explores the use of interference-canceling matrices that are multiplied by user signals to enhance future wireless communications for devices that have limited processing abilities (i.e, mMTC and IoT devices). The system is designed such that there simultaneous transmissions of the same signal from two different antennas. Interferencecanceling matrices are calculated and multiplied by users' signal during each transmission. The downlink transmission from two different antennas is to ensure different channels, consequently enabling the design of the interferencecanceling matrices $\left(\mathbf{P}_{1}, \mathbf{P}_{2}, \mathbf{P}_{3}\right.$, and $\left.\mathbf{P}_{4}\right)$ to guarantee diversity gains and multiplexed communications for minimum resource utilization. The frequency response of each OFDM symbol for user data at Tx1 and Tx2 is represented as $\mathbf{x}(\mathbf{n})=\left[\mathbf{x}_{\mathbf{0}}, \mathbf{x}_{\mathbf{1}}, \ldots, \mathbf{x}_{(N f-1)}\right]$ where $N f$ is the total number of modulated symbols in one OFDM block, and $\mathbf{x}(n) \in$ $C^{[N f \times 1]}$.

Moreover, the channel responses between the transmit and receive antennas are assumed to be known at the transmitter and are considered to be slowly varying multipath Rayleigh fading channels with exponential decay. Also, channel sounding techniques is employed to estimate the channel between the transmitters and receivers. The technique enables the acquisition of the channel from the uplink transmission in a time division duplexing (TDD) system. The proposed paradigm utilizes dual antennas for transmission, and the transmission is done simultaneously.

In Fig. 1, it can be observed that superimposed user signal with interference-canceling matrices are transmitted from two antennas where the transmitted signals are broadcasted to a single dual-antenna user. $\mathbf{P}_{\mathbf{1}}, \mathbf{P}_{\mathbf{2}}, \mathbf{P}_{\mathbf{3}}$, and $\mathbf{P}_{\mathbf{4}}$ are special interference-canceling matrices designed to prevent interstream interference at the receiver.

The basic steps for the design of interference-canceling matrices for the proposed algorithm are presented in the subsequent discussions. The superimposed transmitted signal with interference-canceling matrices from $\mathrm{Tx} 1$ is given as:

$$
\mathbf{u}_{1}=\mathbf{P}_{1} \mathbf{x}_{1}+\mathbf{P}_{2} \mathbf{x}_{2}
$$


Similarly, the superimposed transmitted signal with interference-canceling matrices from $\mathrm{T} \times 2$ is given as:

$$
\mathbf{u}_{2}=\mathbf{P}_{3} \mathbf{x}_{1}+\mathbf{P}_{4} \mathbf{x}_{2},
$$

where $\mathrm{x}_{1}$ and $\mathrm{x}_{\mathbf{2}}$ are data vectors in frequency domain intended for the user, with the total power divided equally between them, while $\mathbf{P}_{\mathbf{1}}, \mathbf{P}_{\mathbf{2}}, \mathbf{P}_{\mathbf{3}}$, and $\mathbf{P}_{\mathbf{4}}$ are specially designed interference-canceling matrices based on the channel of authorized nodes. The interference-canceling matrices designed at the base station will remove inter-symbol interference at the receiver without any processing, this is the main reason why the proposed system is best suited for mMTC and IoT devices. The details about the received signal at $\mathrm{Rx} 1$ and $\mathrm{Rx} 2$ will first be explained in the following subsections. Afterward, the details about designing the interferencecanceling matrices will be explained.

\section{A. RECEIVED SIGNAL AT RECEIVER ANTENNA 1 (RX1)}

The received signal in the frequency domain at $\mathrm{Rx} 1$ from $\mathrm{Tx} 1$ is given as:

$$
\mathbf{y}_{11}=\mathbf{H}_{11} \mathbf{u}_{1}
$$

where $\mathbf{H}_{11}$ is the frequency response of the channel between Rx1 and transmitting antenna Tx1. Similarly, the received signal at $\mathrm{Rx} 1$ from transmission using $\mathrm{T} \times 2$ is given as:

$$
\mathrm{y}_{12}=\mathrm{H}_{12} \mathbf{u}_{2}
$$

where $\mathbf{H}_{12}$ is the frequency response of the channel, between $\mathrm{Rx} 1$ and transmitting antenna $\mathrm{Tx} 2$. The combined received signal at Rx1 by simultaneous transmission from $\mathrm{Tx} 1$ and $\mathrm{Tx} 2$ is given as:

$$
\hat{\mathbf{y}}_{1}=\mathbf{y}_{11}+\mathbf{y}_{12}+\mathbf{z}_{1},
$$

where $\mathbf{z}_{1}$ is the additive white Gaussian noise (AWGN) at Rx1. Substituting the values of $\mathbf{y}_{11}$ and $\mathbf{y}_{12}$, the combined signal is written as:

$$
\hat{\mathbf{y}}_{1}=\mathbf{H}_{11} \mathbf{u}_{1}+\mathbf{H}_{12} \mathbf{u}_{2}+\mathbf{z}_{1}
$$

Substituting the values of $\mathbf{u}_{\mathbf{1}}$ and $\mathbf{u}_{\mathbf{2}}$ from (2) and (3) and simplifying, results in:

$$
\hat{\mathbf{y}}_{1}=\mathbf{H}_{11}\left(\mathbf{P}_{1} \mathbf{x}_{1}+\mathbf{P}_{2} \mathbf{x}_{2}\right)+\mathbf{H}_{12}\left(\mathbf{P}_{3} \mathbf{x}_{1}+\mathbf{P}_{4} \mathbf{x}_{2}\right)+\mathbf{z}_{1},
$$

Simplifying (8) results in:

$$
\hat{\mathbf{y}}_{\mathbf{1}}=\left(\mathbf{H}_{11} \mathbf{P}_{\mathbf{1}}+\mathbf{H}_{12} \mathbf{P}_{\mathbf{3}}\right) \mathbf{x}_{\mathbf{1}}+\left(\mathbf{H}_{11} \mathbf{P}_{\mathbf{2}}+\mathbf{H}_{12} \mathbf{P}_{4}\right) \mathbf{x}_{\mathbf{2}}+\mathbf{z}_{\mathbf{1}} \text { (9) }
$$

The first term in (9) is the desired term with respect to Rx1, while the remaining terms are undesired. The interferencecanceling matrices will make sure that the undesired term as well as the channel effects are completely removed at Rx1.

\section{B. RECEIVED SIGNAL AT RECEIVER ANTENNA 2 (RX2)}

Similarly, the expression for the combined received signal at $\mathrm{Rx} 2$ by simultaneous transmission through $\mathrm{Tx} 1$ and $\mathrm{Tx} 2$ is given as:

$$
\hat{\mathbf{y}}_{2}=\mathrm{y}_{21}+\mathrm{y}_{22}+\mathrm{z}_{2},
$$

where $\mathbf{y}_{21}=\mathbf{H}_{21} \mathbf{u}_{1}$, and $\mathbf{y}_{22}=\mathbf{H}_{22} \mathbf{u}_{2}$ are the received signals at $\mathrm{Rx} 2$ from $\mathrm{Rx} 1$ and $\mathrm{Rx} 2$ respectively and $\mathrm{z}_{2}$ is the AWGN at Rx2. $\mathbf{H}_{21}$ is the frequency response of the channel between $\mathrm{Rx} 2$ and $\mathrm{Tx} 1$, while $\mathbf{H}_{\mathbf{2} 2}$ is the frequency response of the channel between Rx2 and Tx2. After substituting the values of $\mathbf{y}_{21}$ and $\mathbf{y}_{22}$, the combined signal is presented as:

$$
\hat{\mathbf{y}}_{2}=\mathbf{H}_{21} \mathbf{u}_{1}+\mathbf{H}_{22} \mathbf{u}_{2}+\mathbf{z}_{2} .
$$

Substituting the values of $\mathbf{u}_{1}$ and $\mathbf{u}_{\mathbf{2}}$ from (2) and (3) to (11) results in (12).

$$
\hat{\mathbf{y}}_{2}=\mathbf{H}_{21}\left(\mathbf{P}_{1} \mathbf{x}_{1}+\mathbf{P}_{2} \mathbf{x}_{2}\right)+\mathbf{H}_{22}\left(\mathbf{P}_{3} \mathbf{x}_{1}+\mathbf{P}_{4} \mathbf{x}_{2}\right)+\mathbf{z}_{2} .
$$

Simplifying (12) results in (13).

$$
\left.\hat{\mathbf{y}}_{\mathbf{2}}=\left(\mathbf{H}_{21} \mathbf{P}_{1}+\mathbf{H}_{22} \mathbf{P}_{3}\right) \mathbf{x}_{1}+\left(\mathbf{H}_{21} \mathbf{P}_{\mathbf{2}}+\mathbf{H}_{22} \mathbf{P}_{\mathbf{4}}\right) \mathbf{x}_{\mathbf{2}}+\mathbf{z}_{\mathbf{2}} 13\right)
$$

The first term of (13) is the undesired term with respect to $\mathrm{Tx} 2$, while the second term is desired.

\section{DESIGNING THE INTERFERENCE-CANCELING MATRICES}

The interference-canceling matrices $\mathbf{P}_{\mathbf{1}}, \mathbf{P}_{\mathbf{2}}, \mathbf{P}_{\mathbf{3}}$, and $\mathbf{P}_{\mathbf{4}}$ are designed in such a way that the matrices will be multiplied by user data at the base station before transmission and upon reception the matrices will prevent inter-symbol interference without any processing at the receiver. The design procedure of $\mathbf{P}_{\mathbf{1}}$ and $\mathbf{P}_{\mathbf{3}}$ is as follows: Firstly, in order to remove the effect of the channel at Rx1, the first term in (9) should be equal to an identity matrix given as:

$$
\mathbf{H}_{11} \mathbf{P}_{1}+\mathbf{H}_{12} \mathbf{P}_{3}=\mathbf{I} .
$$

Also, in order to cancel the interference caused by the signal for $\mathrm{Rx} 1$ on the signal for $\mathrm{Rx} 2$, the first term in equation (13) should be zero and is given as:

$$
\mathbf{H}_{21} \mathbf{P}_{1}+\mathbf{H}_{22} \mathbf{P}_{3}=\mathbf{0} .
$$

Equations (14) and (15) can be jointly solved to get the values of interference-canceling matrices $\mathbf{P}_{\mathbf{1}}$ and $\mathbf{P}_{\mathbf{3}}$ as follows:

$$
\begin{gathered}
\mathbf{P}_{1}=\frac{-\mathbf{H}_{22}}{\left(\mathbf{H}_{12} \mathbf{H}_{21}-\mathbf{H}_{11} \mathbf{H}_{22}\right)} . \\
\mathbf{P}_{3}=\frac{\mathbf{H}_{21}}{\left(\mathbf{H}_{12} \mathbf{H}_{21}-\mathbf{H}_{11} \mathbf{H}_{22}\right)} .
\end{gathered}
$$

Similarly, in order to design $\mathbf{P}_{\mathbf{2}}$ and $\mathbf{P}_{\mathbf{4}}$, similar steps will be followed as explained earlier. To remove the effect of the 
channel at $\mathrm{Tx} 2$, the second term in equation (13) should be equal to identity matrix and is given as:

$$
\mathbf{H}_{21} \mathbf{P}_{2}+\mathbf{H}_{22} \mathbf{P}_{4}=\mathbf{I} \text {. }
$$

Also, in order to cancel the interference caused by the signal for Rx2 on the signal for Rx1, the second term in (9) should be zero, given as:

$$
\mathbf{H}_{11} \mathbf{P}_{2}+\mathbf{H}_{12} \mathbf{P}_{4}=\mathbf{0}
$$

Equations (18) and (19) can be jointly solved to get the values of interference-canceling matrices as follows:

$$
\begin{gathered}
\mathbf{P}_{2}=\frac{-\mathbf{H}_{12}}{\left(\mathbf{H}_{11} \mathbf{H}_{22}-\mathbf{H}_{12} \mathbf{H}_{21}\right)} . \\
\mathbf{P}_{4}=\frac{\mathbf{H}_{11}}{\left(\mathbf{H}_{11} \mathbf{H}_{22}-\mathbf{H}_{12} \mathbf{H}_{21}\right)} .
\end{gathered}
$$

The values of interference-canceling matrices $\mathbf{P}_{\mathbf{1}}, \mathbf{P}_{\mathbf{2}}, \mathbf{P}_{\mathbf{3}}$, and $\mathbf{P}_{4}$ given in equations (16), (20), (17), and (21) respectively, will be used in the simultaneous transmission of superimposed user data to make sure that the user will get reliable signals.

\section{APPROACH TO PERFORMANCE ANALYSIS}

In order to conduct the performance analysis on the proposed MIMO technique, data analysis models for the user will be designed and discussed in the following sections. Due to the use of the interference-canceling matrices practical and ideal models will be analysed. In the ideal setting power may not be a limiting factor, however, in practical implementations transmit power is a big factor. Analysing the proposed model from the ideal point of view (as observed in section III), it can be concluded that using interference-canceling matrices will cancel both the interference and channel effects, hence leading to AWGN performance $(y=x+$ noise). However, in practical scenarios, this is not the case, due to the use of the matrices the signal must be normalized before transmission because the power cannot go to infinity, therefore, this will cause some changes as it will be observed in the simulation results section below.

\section{A. PERformanCE AT THE RECEIVER Rx}

Numerical data fitting methods will be used to conduct the performance analysis [30]. Moreover, instantaneous signalto-noise ratio $\gamma_{b}$ at each receiver antenna is calculated to find their BER. Authors in [30] further stated that in order to calculate $\gamma_{b}$ using numeric data fitting methods, the power distribution of sub-channels corresponding to each of the receiver antenna ( $\mathrm{Rx} 1$ and $\mathrm{Rx} 2)$ must be calculated. In this model, the power distribution of the sub-channels corresponding to the received signal on Rx is depicted in Fig. 3.

The theoretical BER of the proposed MIMO model is calculated using the analytical data fitting method described in [30]. First, the effective instantaneous signal-to-noise ratio
(SNR), $\gamma_{b}$ is determined, then the probability density function for the effective instantaneous SNR is calculated as:

$$
P_{\gamma b}\left(\gamma_{b}\right) \approx\left(\frac{1}{\omega}\right)^{\mu} \frac{1}{\Gamma(\mu)} \frac{\Omega^{\frac{3}{2}} \sqrt{\gamma_{b}}}{\hat{\gamma}_{b}^{\frac{3}{2}}} \exp \left(-\frac{1}{\omega} \frac{\omega \gamma_{b}}{\hat{\gamma}_{b}}\right),
$$

where $\Omega, \hat{\gamma}_{b}$, and $\Gamma(\mu)$ are the mean squire of the subchannels, average SNR, and the gamma function respectively. $P_{\gamma b}\left(\gamma_{b}\right)$ is then used to calculate the BER.

$$
B E R_{b}=\frac{1}{2} \int_{0}^{\infty} \operatorname{erfc}\left(\sqrt{\gamma_{b}}\right) P_{\gamma_{b}}\left(\gamma_{b}\right) d \gamma_{b} .
$$

Substituting $P_{\gamma b}\left(\gamma_{b}\right)$ in (23) with (22) results in (28).

$$
B E R_{b}=\frac{1}{2} \int_{0}^{\infty} \operatorname{erfc}\left(\sqrt{\gamma_{b}}\right)\left(\frac{1}{\omega}\right)^{\mu} \frac{1}{\Gamma(\mu)} \frac{\Omega^{\frac{3}{2}} \sqrt{\gamma_{b}}}{\frac{\gamma_{b}^{3}}{\gamma_{b}^{2}}} \exp \left(-\frac{1}{\omega} \frac{\omega \gamma_{b}}{\hat{\gamma}_{b}}\right) d \gamma_{b} .
$$

According to [31] simplifying (28) results in (29).

$$
B E R_{b} \approx \frac{\left(\frac{1}{\omega}\right)^{\mu} \frac{1}{\Gamma(\mu)} \frac{\Omega^{\frac{3}{2}}}{\hat{\gamma}_{b}^{\frac{3}{2}}}}{2 \sqrt{\pi}}\left(\frac{\arctan \left(\sqrt{\frac{1}{\omega} \frac{\omega}{\hat{\gamma}_{b}}}\right)}{2 \frac{1}{\omega} \frac{\omega}{\hat{\gamma}_{b}} 3 / 2}-\frac{1}{2 \frac{1}{\omega} \frac{\omega}{\hat{\gamma}_{b}}\left(1+\frac{1}{\omega} \frac{\Omega}{\hat{\gamma}_{b}}\right)}\right),
$$

where, $\arctan (\cdot)$ denotes the inverse tangent.

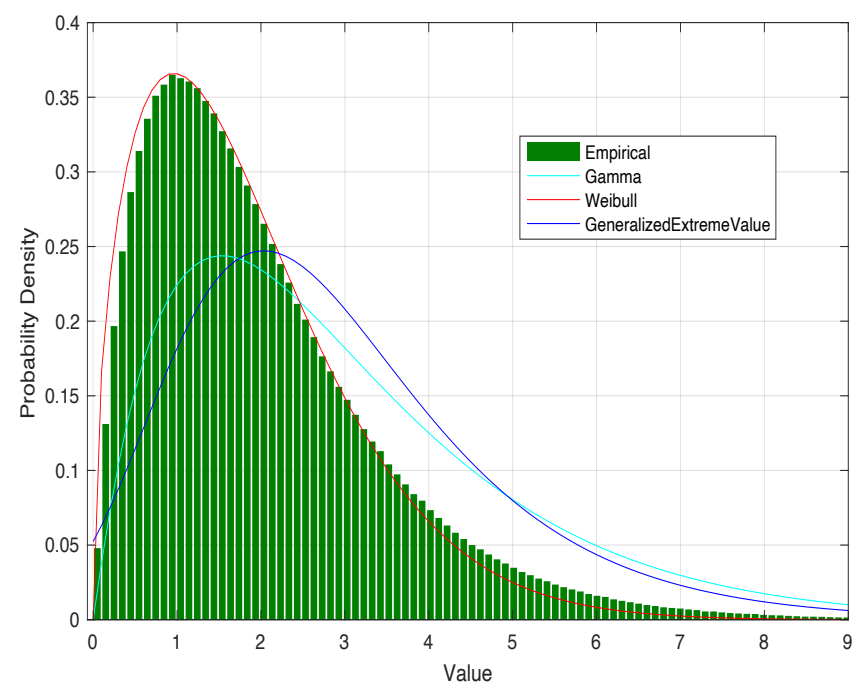

FIGURE 3. Power distribution of sub-channels corresponding to the received signal at legitimate user (Bob)

\section{SIMULATION RESULTS AND DISCUSSIONS}

In this section, the performance metrics of the proposed algorithm will be analyzed in terms of throughput, packet error rate (PER), bit error rate (BER), and more. The specifications of the simulated model are given in Table 1.

From the Table, it can be observed that the channels between all MIMO transmitters and receivers are assumed to be slowly fading Rayleigh with equal number of taps $(L=9)$. Also, the system uses OFDM transmitters for each antenna (Tx1 and Tx2) with 64 sub-carrier for each receiver. 
TABLE 1. Proposed algorithm system parameters

\begin{tabular}{|l|l|}
\hline Channel & Multipath Rayleigh Fading Channel \\
\hline Channel Length (L) & 9 \\
\hline Cyclic Prefix (CP) & 9 \\
\hline FFT Size & 64 \\
\hline Modulation Type & BPSK \\
\hline
\end{tabular}

Moreover, a cyclic prefix of length 9 is added to help mitigate inter-symbol interference (ISI).

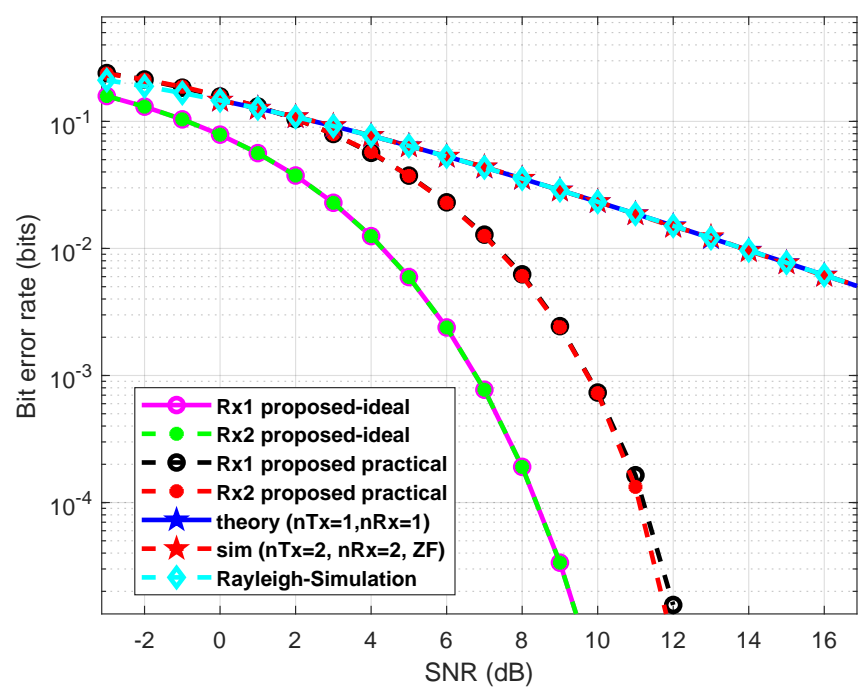

FIGURE 4. Bit error rate (BER) of the proposed algorithm depicting the ideal and practical case, moreover, a comparison with other conventional models such 2 by 2 zero-forcing MIMO, theoretical SISO model, and MIMO under Rayleigh simulation .

Fig. 4 shows the BER of the proposed algorithm in comparison with a conventional MIMO models. Rx1 proposed - ideal shows the BER at the first receiver antenna while $R x 2$ - proposed - ideal is the BER at the second receiver antenna. The figure also depicts the theoretical BER of a single-input single-output (SISO) model (theory $(\mathrm{nTx}=1, \mathrm{nRx}=1))$ and a two-output two-input MIMO simulation model with zero forcing (ZF) $(\operatorname{sim}(\mathrm{nTx}=2, \mathrm{nRx}=2, \mathrm{ZF}))$ as well as Rayleigh simulation. $R x 1$ - proposed-practical and $R x 2$ - proposed-practical are the practical BER plots of the proposed model.

As it can be observed, the proposed MIMO model perform better than conventional MIMO model utilizing ZF, the SISO model, and the Rayleigh simulation performance curve. Moreover, the proposed ideal model exhibits AWGN performance, this is due to the utilization of the interferencecanceling matrices $\left(\mathbf{P}_{1}, \mathbf{P}_{2}, \mathbf{P}_{3}, \mathbf{P}_{4}\right)$ which are designed to cancel interference as well as channel effects. However, the practical case shows degraded BER performance because the transmitted signal that contains the interference-canceling matrices must be normalized due to power limitations, hence degrading the signal. The interference-canceling matrices are functions of the Rayleigh channel used to simulate this model as shown in section III. Since Rayleigh channel distribution takes values very close to zero, performing division with these values to get the values of the interference-canceling matrices (the power) will result to a very large value (infinity), this is not practical hence the normalization.

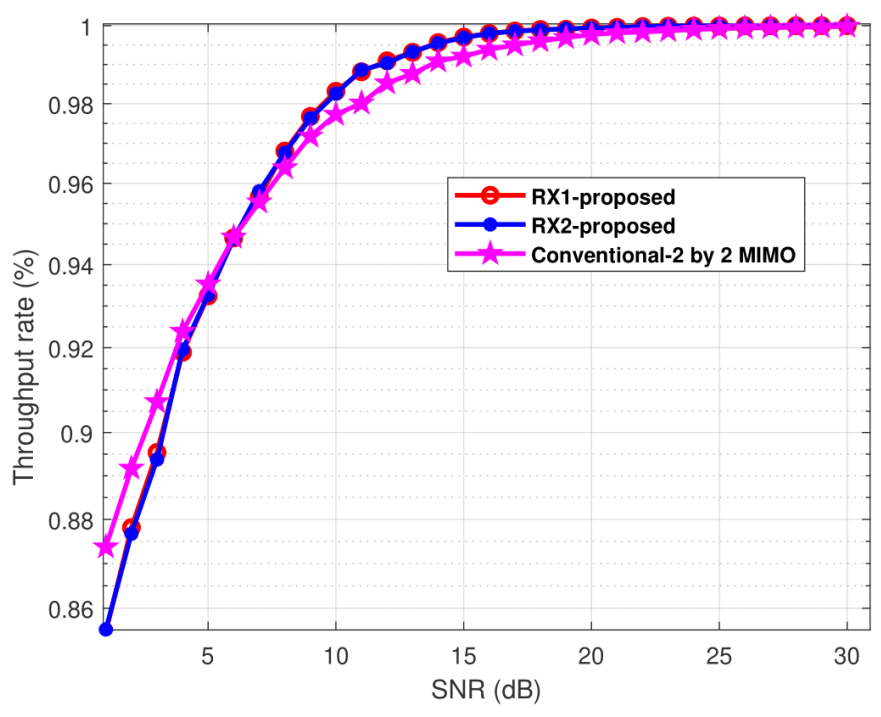

FIGURE 5. Throughput of the proposed MIMO model in comparison with a conventional 2 by 2 MIMO system with no interferance-canceling matrices.

Fig. 5 depicts the throughput of the proposed model under different signal-to-noise ratio (SNR) values. $R x 1$ - proposed and $R x 2-$ proposed are the throughput of the first and second receiver antennas respectively, while Conventional 2 by $2 M I M O$ is the throughput of a conventional 2 by 2 MIMO system. As it can be observed for $\mathrm{SNR}<8 \mathrm{~dB}$ the proposed model has slightly less throughput, however, for SNR $>8 \mathrm{~dB}$ the proposed model has better throughput.

Fig. 6 shows the peak to average power ratio (PAPR) of a system utilizing the proposed MIMO model compared to a conventional MIMO model. It can be observed that the PAPR of the two transmit antennas (Tx1-proposed and Tx2proposed) utilizing the proposed paradigm is slightly better than the PAPR of transmit antennas utilizing a conventional MIMO system (Tx1-conventional and Tx2-conventional). Reduced PAPR leads to better spectral and energy efficiency, therefore, the proposed MIMO model solves one of the major problems experienced by OFDM systems [32].

\section{ADVANTAGES OF THE PROPOSED SYSTEM}

1) Low complexity: It is structurally simple but very effective, and it does not require to be supported by a complicated transceiver architectures. More importantly, it does not require any changes or extra processing at the receiver side thanks to the proper design of the added interference-canceling matrices.

2) Low power: Since no processing is required at the receiver, there is no use of high-power during communication which usually is a big consumer of energy in 


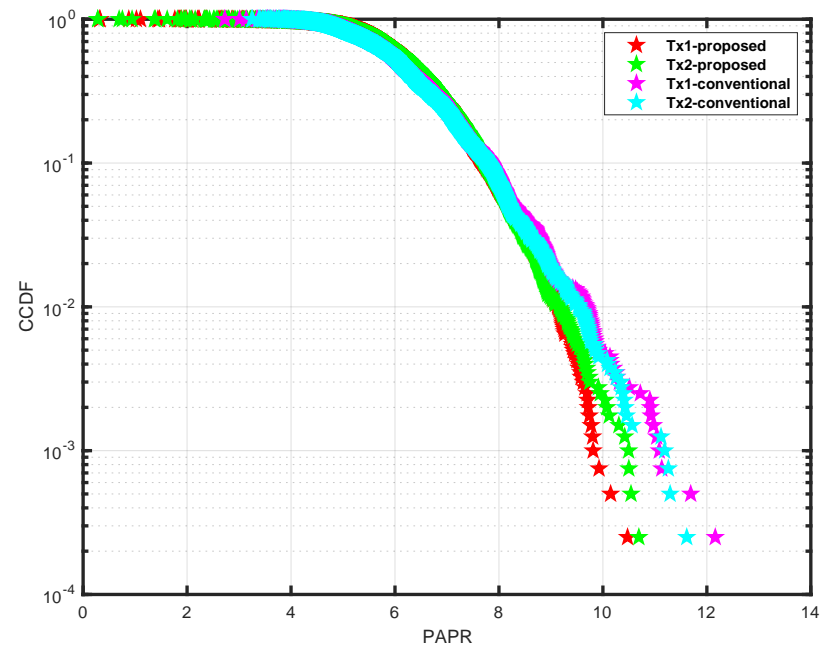

FIGURE 6. Peak to average power ratio (PAPR) of the proposed algorithm compared to a conventional model. The figure indicates that for lower complementary cumulative distribution function (CCDF) values the proposed model performs slightly better than conventional MIMO methods

conventional systems. All the processing is done at the base station.

3) The maximum benefit and best operating condition of the proposed scheme can be obtained when it is used with OFDM-based waveforms over dispersive channels. This is due to two reasons: 1) the interferencecanceling matrices' $\left(\mathbf{P}_{1}, \mathbf{P}_{\mathbf{2}}, \mathbf{P}_{\mathbf{3}}\right.$, and $\left.\mathbf{P}_{4}\right)$ randomness becomes not only a function of the generated signal at the source but also of the dispersive channel randomness. 2) the possibility of redesigning the matrices to solve some of the major drawbacks of OFDM.

\section{LIMITATIONS OF THE STUDY}

The proposed model is designed for downlink communication only. Therefore, a conventional communication technique will be used for uplink communication. Moreover, for a system with many antennas, higher processing power may be required to compute the interference-canceling matrices at the base station.

\section{CONCLUSION AND FUTURE WORKS}

In this work, a novel MIMO model with increased data rate, reduced BER, and beamforming capabilities is proposed. The model is used to achieve diversity and multiplexing gains. Interference-canceling matrices $\mathbf{P}_{\mathbf{1}}, \mathbf{P}_{\mathbf{2}}, \mathbf{P}_{\mathbf{3}}$, and $\mathbf{P}_{\mathbf{4}}$ are specially designed from channel characteristics and multiplied by the user data before superposition and simultaneous transmission from the two transmit antennas. Simulation results indicate that the proposed MIMO model outperforms conventional systems and hence suitable for next-generation wireless communication applications, specifically IoT and mMTC devices. In the future, we will consider increasing the number of antennas in the proposed MIMO model to more than two antennas at both the transmitter and receiver.

\section{REFERENCES}

[1] J. P. Lemayian and J. M. Hamamreh, "Recurrent neural network-based channel prediction in mMIMO for enhanced performance in future wireless communication," in 2020 International Conference on UK-China Emerging Technologies (UCET). IEEE, 2020, pp. 1-4.

[2] — " "Massive MIMO channel prediction using recurrent neural networks," RS Open Journal on Innovative Communication Technologies, vol. 1 , no. 1,82020 .

[3] T. N. Do and Z. J. Haas, "On the design of RD-MIMO: Spatial multiplexing versus opportunistic transmission schemes," IEEE Access, vol. 8, pp $22733-22747,2020$

[4] S. Nandi, N. N. Pathak, and A. Nandi, "A novel adaptive optimized fas blind channel estimation for cyclic prefix assisted space-time block coded MIMO-OFDM systems," Wireless Personal Communications, vol. 115 no. 2, pp. 1317-1333, 2020.

[5] A. PramodKumar, N. Suman, Y. Koteswararao, and D. Venkatachari, "Design and analysis of high beam forming MIMO antenna for 5G applications," Materials Today: Proceedings, 2020.

[6] W. Saad, M. Bennis, and M. Chen, "A vision of $6 \mathrm{G}$ wireless systems: Applications, trends, technologies, and open research problems," IEEE network, vol. 34, no. 3, pp. 134-142, 2019.

[7] J. P. Lemayian and J. M. Hamamreh, "A novel small-scale nonorthogonal communication technique using auxiliary signal superposition with enhanced security for future wireless networks," RS Open Journal on Innovative Communication Technologies, vol. 1, no. 2, 112020.

[8] J. M. Hamamreh, M. Abewa, and J. P. Lemayian, "New non-orthogonal transmission schemes for achieving highly efficient, reliable, and secure multi-user communications," RS Open Journal on Innovative Communication Technologies, vol. 1, no. 2, 122020.

[9] J. M. Hamamreh and H. Arslan, "Joint PHY/MAC layer security design using ARQ with MRC and null-space independent PAPR-aware artificial noise in SISO systems," IEEE Transactions on Wireless Communications, vol. 17, no. 9, pp. 6190-6204, 2018.

[10] - "Secure orthogonal transform division multiplexing (OTDM) waveform for $5 \mathrm{G}$ and beyond," IEEE Communications Letters, vol. 21, no. 5, pp. 1191-1194, 2017.

[11] M. R. Amin and S. D. Trapasiya, "Performance optimization of MIMO using space-time block coding scheme," Procedia engineering, vol. 38, pp 3518-3527, 2012.

[12] J. C. Horng and C. Ngo, "Combining space time block code (STBC) with spatial multiplexing for MIMO transmission," Feb. 16 2010, uS Patent $7,664,194$.

[13] L. Zhao and V. K. Dubey, "Detection schemes for space-time block code and spatial multiplexing combined system," IEEE Communications Letters, vol. 9, no. 1, pp. 49-51, 2005.

[14] Y. Wu and C. Tellambura, "Low-complexity optimal detection for hybrid space-time block coding and spatial multiplexing," in IEEE Vehicular Technology Conference. IEEE, 2006, pp. 1-4.

[15] N. Al-Dhahir, "A new high-rate differential space-time block coding scheme," IEEE Communications Letters, vol. 7, no. 11, pp. 540-542, 2003

[16] Y. Li, N. R. Sollenberger, and J. H. Winters, "MIMO OFDM system," Jun. 27 2006, uS Patent 7,068,628.

[17] T. Fukagawa, Y. Nakagawa, and M. Ukena, "MIMO communication device," Jun. 26 2012, uS Patent 8,208,981.

[18] Q. Li and X. E. Lin, "MIMO communication system and method for beamforming using polar-cap codebooks," Feb. 8 2011, uS Patent 7,885,348.

[19] H. Harel, E. Abreu, and K. Kludt, "Implementing blind tuning in hybrid MIMO RF beamforming systems," Feb. 4 2014, uS Patent 8,644,413.

[20] M. X. Gong and R. Stacey, "Systems and methods for uplink multi-user multiple input multiple output (MU MIMO) medium access and error recovery," Nov. 6 2012, uS Patent 8,306,010.

[21] H. J. Song, A. Bekaryan, J. H. Schaffner, E. Yasan, D. S. Carper, and T. J. Talty, "Method of maximizing MIMO throughput by placing antennas in a vehicle," Feb. 14 2017, uS Patent 9,571,172.

[22] K. Kludt, E. Abreu, and H. Harel, "Using antenna pooling to enhance a MIMO receiver augmented by RF beamforming," Feb. 11 2014, uS Patent $8,649,458$.

[23] S. Ashrafi and R. D. Linquist, "System and method for combining MIMO and mode-division multiplexing," Jun. 12 2018, uS Patent 9,998,187.

[24] Q. Li and X. Lin, "Techniques to time vary pilot locations in wireless networks," May 12 2009, uS Patent 7,532,675.

[25] M. El-Hajjar and L. Hanzo, "Multifunctional MIMO systems: A combined diversity and multiplexing design perspective,' IEEE Wireless Communications, vol. 17 , no. 2 , pp. 73-79, 2010 
[26] V. Tarokh, A. Naguib, N. Seshadri, and A. R. Calderbank, "Combined array processing and space-time coding," IEEE Transactions on Information Theory, vol. 45, no. 4, pp. 1121-1128, 1999.

[27] G. Jongren, M. Skoglund, and B. Ottersten, "Combining beamforming and orthogonal space-time block coding," IEEE Transactions on Information theory, vol. 48, no. 3, pp. 611-627, 2002.

[28] J. Liu and E. Gunawan, "Combining ideal beamforming and Alamouti space-time block codes," Electronics letters, vol. 39, no. 17, pp. 1258$1259,2003$.

[29] L.-L. Yang and L. Hanzo, "Performance of generalized multicarrier DSCDMA over nakagami-m fading channels," IEEE Transactions on Communications, vol. 50, no. 6, pp. 956-966, 2002.

[30] J. M. Hamamreh, E. Basar, and H. Arslan, "OFDM-subcarrier index selection for enhancing security and reliability of 5G URLLC services," IEEE Access, vol. 5, pp. $25863-25$ 875, 2017.

[31] Y. Yuan and C. Yan, "NOMA study in 3GPP for 5G," in 2018 IEEE 10th International Symposium on Turbo Codes \& Iterative Information Processing (ISTC). IEEE, 2018, pp. 1-5.

[32] I. Baig, N. ul Hasan, M. Zghaibeh, I. U. Khan, and A. S. Saand, "A DST precoding based uplink NOMA scheme for PAPR reduction in $5 \mathrm{G}$ wireless network," in 2017 7th International Conference on Modeling, Simulation, and Applied Optimization (ICMSAO). IEEE, 2017, pp. 1-4.

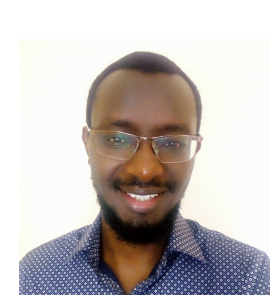

JOEL P. LEMAYIAN Received the B.Sc. degree in electrical and electronics engineering from Middle East Technical University Turkey in 2017, and the M.Sc. degree in electrical and computer engineering from Antalya Bilim University Turkey in 2021. He is currently a research assistant at Antalya Bilim University, Turkey at the wireless communication laboratory.

He has worked as a research assistant in both Middle East Technical University and Antalya Bilim University in the Internet of Things (IoT) lab and Neuroscience lab respectively. He is an author of numerous journals, conference papers, and book chapters. His research interests include physical layer security, 5G communication networks, Artificial Intelligence, Machine Learning, and IoT and its applications.

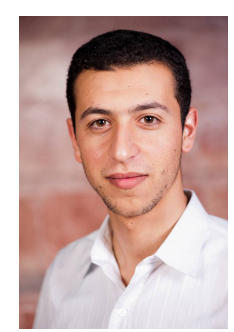

JEHAD M. HAMAMREH is currently an Assistant Professor with the Electrical and Electronics Engineering Department, Antalya International (Bilim) University, Turkey. He received the Ph.D. degree in electrical-electronics engineering and cyber systems from Istanbul Medipol University, Turkey, in 2018. Previously, He worked as a Researcher at the Department of Electrical and Computer Engineering in Texas AM University. He is the inventor of $10+$ Patents, and has authored more than $55+$ peer reviewed scientific papers along with several book chapters. His innovative patented works won the golden, silver and bronze medals by numerous international invention contests and fairs.

His current research interests include wireless physical and MAC layers security, orthogonal frequency-division multiplexing multiple-input multiple-output systems, advanced waveforms design, multidimensional modulation techniques, and orthogonal/non-orthogonal multiple access schemes for future wireless systems. He is a regular investigator and referee for various scientific journals as well as a TPC member for several international conferences. He is an Editor at RS-OJICT and Frontiers in Communications and Networks. 